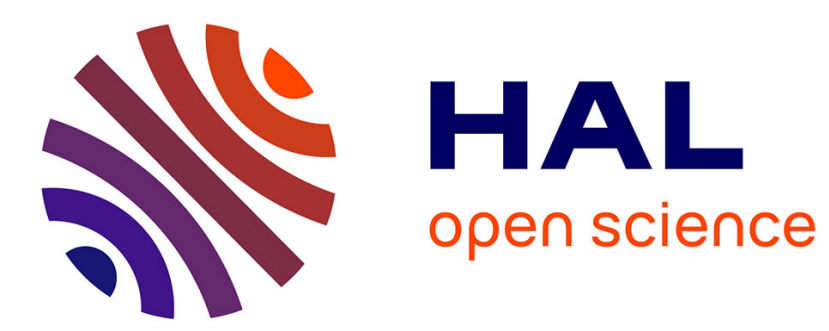

\title{
ON THE COHOMOLOGY OF THE AFFINE SPACE
}

\author{
Wieslawa Niziol, Pierre Colmez
}

\section{To cite this version:}

Wieslawa Niziol, Pierre Colmez. ON THE COHOMOLOGY OF THE AFFINE SPACE. p-adic Hodge

Theory. Simons Symposia., pp.71-80, 2020, 10.1007/978-3-030-43844-9_2 . ensl-01663178v2

\section{HAL Id: ensl-01663178 \\ https://hal-ens-lyon.archives-ouvertes.fr/ensl-01663178v2}

Submitted on 14 Dec 2020

HAL is a multi-disciplinary open access archive for the deposit and dissemination of scientific research documents, whether they are published or not. The documents may come from teaching and research institutions in France or abroad, or from public or private research centers.
L'archive ouverte pluridisciplinaire HAL, est destinée au dépôt et à la diffusion de documents scientifiques de niveau recherche, publiés ou non, émanant des établissements d'enseignement et de recherche français ou étrangers, des laboratoires publics ou privés. 


\title{
ON THE COHOMOLOGY OF THE AFFINE SPACE
}

\author{
by
}

Pierre Colmez \& Wiesława Nizioł

\begin{abstract}
We compute the p-adic geometric pro-étale cohomology of the rigid analytic affine space (in any dimension). This cohomology is non-zero, contrary to the étale cohomology, and can be described by means of differential forms.
\end{abstract}

\section{Introduction}

Let $K$ be a complete discrete valuation field of characteristic 0 with perfect residue field of positive characteristic $p$. Let $C$ be the completion of an algebraic closure $\bar{K}$ of $K$. We denote by $\mathscr{G}_{K}$ the absolute Galois group of $K$ (it is also the group of continuous automorphisms of $C$ that fix $K$ ).

For $n \geq 1$, let $\mathbf{A}_{K}^{n}$ be the rigid analytic affine space over $K$ of dimension $n$ and $\mathbf{A}^{n}$ be its scalar extension to $C$. Our main result is the following theorem.

Theorem 1. - For $r \geq 1$, we have isomorphisms of $\mathscr{G}_{K}$-Fréchet spaces

$$
H_{\text {proét }}^{r}\left(\mathbf{A}^{n}, \mathbf{Q}_{p}(r)\right) \simeq \Omega^{r-1}\left(\mathbf{A}^{n}\right) / \operatorname{Ker} d \simeq \Omega^{r}\left(\mathbf{A}^{n}\right)^{d=0},
$$

where $\Omega$ denotes the sheaf of differentials.

Remark 2. - (i) The p-adic pro-étale cohomology behaves in a remarkably different way from other (more classical) cohomologies. For example, for $i \geq 1$, we have :

- $H_{\mathrm{dR}}^{i}\left(\mathbf{A}^{n}\right)=H_{\mathrm{HK}}^{i}\left(\mathbf{A}^{n}\right)=0$, where $H_{\mathrm{HK}}^{\bullet}$ is Hyodo-Kato cohomology (see [5] for its definition),

- $H_{\text {êt }}^{i}\left(\mathbf{A}^{n}, \mathbf{Q}_{\ell}\right)=H_{\text {proét }}^{i}\left(\mathbf{A}^{n}, \mathbf{Q}_{\ell}\right)=0$, if $\ell \neq p$,

- $H_{\text {ét }}^{i}\left(\mathbf{A}^{n}, \mathbf{Q}_{p}\right)=0$. (Cf. [1] or Remark 12.)

We listed the $\ell \neq p$ and $\ell=p$ cases of étale cohomology separately because, if $\ell \neq p$, the triviality of the cohomology of $\mathbf{A}^{n}$ is a consequence of the triviality of the cohomology of the closed ball (which explains why the pro-étale cohomology is also trivial), but the $p$-adic étale cohomology of the ball is highly nontrivial. 
(ii) Using overconvergent syntomic cohomology allows to prove a more general result [2, Th. 1.8]: if $X$ is a Stein space over $K$ admitting a semistable model over the ring of integers of $K$, there exists an exact sequence

$$
0 \rightarrow \Omega^{r-1}(X) / \operatorname{Ker} d \rightarrow H_{\text {proét }}^{r}\left(X, \mathbf{Q}_{p}(r)\right) \rightarrow\left(\mathrm{B}_{\mathrm{st}}^{+} \widehat{\otimes} H_{\mathrm{HK}}^{r}(X)\right)^{N=0, \varphi=p^{r}} \rightarrow 0 .
$$

However making syntomic cohomology overconvergent is technically demanding and the simple proof below uses special features of the geometry of the affine space.

(iii) Another possible approach (cf. [6]) is to compute the pro-étale cohomology of the relative fundamental exact sequence $0 \rightarrow \mathbf{Q}_{p}(r) \rightarrow \mathbb{B}_{\text {cris }}^{\varphi=p^{r}} \rightarrow \mathbb{B}_{\mathrm{dR}} / F^{r} \rightarrow 0$.

Let $\stackrel{\circ}{\mathbf{B}}^{n}$ be the open unit ball of dimension $n$. An adaptation of the proof of Theorem 1 shows the following result:

Theorem 3. - For $r \geq 1$, we have isomorphisms of $\mathscr{G}_{K}$-Fréchet spaces

$$
H_{\text {proét }}^{r}\left(\stackrel{\circ}{\mathbf{B}}^{n}, \mathbf{Q}_{p}(r)\right) \simeq \Omega^{r-1}\left(\stackrel{\circ}{\mathbf{B}}^{n}\right) / \operatorname{Ker} d \simeq \Omega^{r}\left(\stackrel{\circ}{\mathbf{B}}^{n}\right)^{d=0} \text {. }
$$

Acknowledgements. - We would like to thank the referee for a careful reading of the manuscript and useful suggestions for improving the exposition.

\section{Syntomic variations}

If $r=1$, one can give an elementary proof of Theorem 1 using Kummer theory, but it does not seem very easy to extend this kind of methods to treat the case $r \geq 2$. Instead we are going to use syntomic methods.

Recall that the étale-syntomic comparison theorem $[7,3]$ reduces the computation of $p$-adic étale cohomology to that of syntomic cohomology ${ }^{(1)}$. The latter is defined as a filtered Frobenius eigenspace of absolute crystalline cohomology (see [4] for a gentle introduction and [7] for a more thorough treatment) and can be thought of as a higher dimensional version of the Fontaine-Lafaille functor. Its computation reduces to a computation of cohomology of complexes built from differential forms, and hence is often doable.

More precisely, if $\mathscr{X}$ is a quasi-compact semistable $p$-adic formal scheme over $\mathscr{O}_{K}$, then the Fontaine-Messing period map [4]

$$
\alpha^{F M}: \tau_{\leq r} \mathrm{R} \Gamma_{\text {syn }}\left(\mathscr{X}_{\mathscr{O}_{C}}, \mathbf{Z}_{p}(r)\right) \rightarrow \tau_{\leq r} \mathrm{R} \Gamma_{\text {ét }}\left(\mathscr{X}_{C}, \mathbf{Z}_{p}(r)\right)
$$

is a $p^{N}$-quasi-isomorphism ${ }^{(2)}$ for a constant $N=N(r)$. This generalizes easily to semistable $p$-adic formal schemes over $\mathscr{O}_{C}$ : the rational étale and pro-étale cohomology of such schemes are computed by the syntomic complexes $\mathrm{R} \Gamma_{\text {syn }}\left(\mathscr{X}_{\mathscr{O}_{C}}, \mathbf{Z}_{p}(r)\right)_{\mathbf{Q}}$

1. The computations in [3] are done over $K$ (or over its finite extensions), but working directly over $C$ simplifies a lot the local arguments because there is no need to change the Frobenius and the group $\Gamma$ of loc. cit. becomes commutative (hence so does its Lie algebra, which makes the arguments using Koszul complexes a lot simpler).

2. It means that the kernel and cokernel of the induced map on cohomology are annihilated by $p^{N}$. 
and $\mathrm{R}_{\mathrm{syn}}\left(\mathscr{X}_{\mathscr{O}_{C}}, \mathbf{Q}_{p}(r)\right)$, respectively, where the latter complex is defined by taking $\mathrm{R} \Gamma_{\text {syn }}\left(\mathscr{X}_{\mathscr{O}_{C}}, \mathbf{Z}_{p}(r)\right)_{\mathbf{Q}}$ locally and then glueing.

The purpose of this section is to construct a particularly simple complex that, morally, computes the syntomic, and hence (pro-)étale as well, cohomology of the (canonical formal model of the) affine space and the open ball, but does not use a model of the whole space, only of closed balls of increasing radii.

Period rings. - Let $C^{b}$ be the tilt of $C$ and let $\mathrm{A}_{\text {cris }} \subset \mathrm{B}_{\text {cris }}^{+}=\mathrm{A}_{\text {cris }}\left[\frac{1}{p}\right] \subset \mathrm{B}_{\mathrm{dR}}^{+}$be the usual Fontaine's rings.

Let $\theta: \mathrm{B}_{\mathrm{dR}}^{+} \rightarrow C$ be the canonical projection (its restriction to $\mathrm{A}_{\text {cris }}$ induces a projection $\mathrm{A}_{\text {cris }} \rightarrow \mathscr{O}_{C}$ ), and let $\mathrm{F}_{\theta} \mathrm{B}_{\mathrm{dR}}^{+}$be the filtration by the powers of $\operatorname{Ker} \theta$ and $\mathrm{F}_{\dot{\theta}} \mathrm{A}_{\text {cris }}$ be the induced filtration. For $j \in \mathbf{Z}$, let $\mathrm{A}_{j}=\mathrm{A}_{\text {cris }} / \mathrm{F}_{\theta}^{j}$ (hence $\mathrm{A}_{j}=0$ for $j \leq 0$ and $\mathrm{A}_{1}=\mathscr{O}_{C}$ ).

We choose a morphism of groups $\alpha \mapsto p^{\alpha}$ from $\mathbf{Q}$ to $C^{*}$ compatible with the analogous morphism on $\mathbf{Z}$. We denote by $\tilde{p}^{\alpha}$ the element $\left(p^{\alpha}, p^{\alpha / p}, p^{\alpha / p^{2}}, \ldots\right)$ of $C^{b}$ and by $\left[\tilde{p}^{\alpha}\right]$ its Teichmüller lift in $\mathrm{A}_{\text {cris }}$.

Closed balls. - For $\alpha \in \mathbf{Q}_{+}$, let

$$
D_{\alpha}=\left\{z=\left(z_{1}, \ldots, z_{n}\right), v_{p}\left(z_{m}\right) \geq-\alpha, \text { for } 1 \leq m \leq n\right\}
$$

be the closed ball of valuation $-\alpha$ in $\mathbf{A}^{n}$, and denote by $\mathscr{O}\left(D_{\alpha}\right)\left(\right.$ resp. $\left.\mathscr{O}^{+}\left(D_{\alpha}\right)\right)$ the ring of analytic functions (resp. analytic functions with integral values) on $D_{\alpha}$. We have

$$
\mathscr{O}\left(D_{\alpha}\right)=C\left\langle p^{\alpha} T_{1}, \ldots, p^{\alpha} T_{n}\right\rangle \quad \text { and } \quad \mathscr{O}^{+}\left(D_{\alpha}\right)=\mathscr{O}_{C}\left\langle p^{\alpha} T_{1}, \ldots, p^{\alpha} T_{n}\right\rangle .
$$

Consider the lifts

$$
R_{\alpha}^{+}=\mathrm{A}_{\text {cris }}\left\langle\left[\tilde{p}^{\alpha}\right] T_{1}, \ldots,\left[\tilde{p}^{\alpha}\right] T_{n}\right\rangle \quad \text { and } \quad R_{\alpha}=R_{\alpha}^{+}\left[\frac{1}{p}\right]
$$

of $\mathscr{O}^{+}\left(D_{\alpha}\right)$ and $\mathscr{O}\left(D_{\alpha}\right)$, respectively. We extend $\varphi$ on $\mathrm{A}_{\text {cris }}$ to $\varphi: R_{\alpha} \rightarrow R_{\alpha}$ by setting $\varphi\left(T_{m}\right)=T_{m}^{p}$, for $1 \leq m \leq n$.

Definition 5. - Let $r \geq 0$. If $\alpha \in \mathbf{Q}_{+}$and $\Lambda=R_{\alpha}, R_{\alpha}^{+}$, we define the complexes

$$
\operatorname{Syn}(\Lambda, r):=\left[\operatorname{HK}_{r}(\Lambda) \rightarrow \operatorname{DR}_{r}(\Lambda)\right],
$$

where the brackets $[\cdots]$ denote the mapping fiber, and ${ }^{(3)}$

$$
\begin{aligned}
\operatorname{HK}_{r}(\Lambda) & :=\left[\Omega_{\Lambda}^{\dot{\varphi-p^{r}}} \rightarrow \Omega_{\Lambda}^{\bullet}\right], \\
F^{r} \Omega_{\Lambda} & :=\left(F_{\theta}^{r} \Lambda \rightarrow F_{\theta}^{r-1} \Omega_{\Lambda}^{1} \rightarrow F_{\theta}^{r-2} \Omega_{\Lambda}^{2} \rightarrow \cdots\right), \\
\operatorname{DR}_{r}(\Lambda) & :=\Omega_{\Lambda} / F^{r}=\left(\cdots \rightarrow \mathrm{A}_{r-i} \otimes_{\mathrm{A}_{\text {cris }}} \Omega_{\Lambda}^{i} \stackrel{1 \otimes d_{i}}{\longrightarrow} \mathrm{A}_{r-i-1} \otimes_{\mathrm{A}_{\text {cris }}} \Omega_{\Lambda}^{i+1} \rightarrow \cdots\right) .
\end{aligned}
$$

3. The differentials are taken relative to $A_{\text {cris }}$. 
The complex $\operatorname{Syn}\left(\mathbf{A}^{n}, r\right)$. - The above complexes for varying $\alpha$ are closely linked:

- The ring morphism $R_{0} \rightarrow R_{\alpha}, T_{m} \rightarrow\left[\tilde{p}^{\alpha}\right] T_{m}$, for $1 \leq m \leq n$, induces an isomorphism of complexes $\operatorname{Syn}\left(R_{0}, r\right) \stackrel{\sim}{\rightarrow} \operatorname{Syn}\left(R_{\alpha}, r\right)$.

- For $\beta \geq \alpha$, the inclusion $\iota_{\beta, \alpha}: R_{\beta} \hookrightarrow R_{\alpha}$ induces a morphism of complexes $\operatorname{Syn}\left(R_{\beta}, r\right) \rightarrow \operatorname{Syn}\left(R_{\alpha}, r\right)$ thanks to the fact that $\varphi\left(\left[\tilde{p}^{s}\right]\right)=\left[\tilde{p}^{s}\right]^{p}$, for all $s \in \mathbf{Q}_{+}$.

(We have analogous statements replacing $R_{\alpha}$ by $R_{\alpha}^{+}$.)

The first point comes just from the fact that two closed balls are isomorphic, but the second point, to the effect that we can find liftings of the $\mathscr{O}\left(D_{\alpha}\right)$ 's with compatible Frobenius, is a bit of a miracle, and will simplify greatly the computation of the syntomic cohomology of $\mathbf{A}^{n}$. In particular, it makes it possible to define the complex $\operatorname{Syn}\left(\mathbf{A}^{n}, r\right):=\operatorname{holim}_{\alpha} \operatorname{Syn}\left(R_{\alpha}, r\right)$ and, similarly, $\operatorname{HK}_{r}\left(\mathbf{A}^{n}\right)$ and $\operatorname{DR}_{r}\left(\mathbf{A}^{n}\right)$.

For $i \geq 0$ and $X=\mathbf{A}^{n}, R_{\alpha}, R_{\alpha}^{+}$, denote by $\operatorname{HK}_{r}^{i}(X), \operatorname{DR}_{r}^{i}(X)$, and $\operatorname{Syn}^{i}(X, r)$ the cohomology groups of the corresponding complexes. We have a long exact sequence:

$$
\cdots \rightarrow \mathrm{DR}_{r}^{i-1}(X) \rightarrow \operatorname{Syn}^{i}(X, r) \rightarrow \operatorname{HK}_{r}^{i}(X) \rightarrow \mathrm{DR}_{r}^{i}(X) \rightarrow \cdots
$$

Proposition 6. - If $i \leq r$, we have natural isomorphisms:

- $H_{\text {ét }}^{i}\left(D_{\alpha}, \mathbf{Q}_{p}(r)\right) \cong \operatorname{Syn}^{i}\left(R_{\alpha}, r\right)$, if $\alpha \in \mathbf{Q}_{+}$.

- $H_{\text {proét }}^{i}\left(\mathbf{A}^{n}, \mathbf{Q}_{p}(r)\right) \cong \operatorname{Syn}^{i}\left(\mathbf{A}^{n}, r\right)$.

Proof. - Take $\alpha \in \mathbf{Q}_{+}$. By the comparison isomorphism (4), to prove the first claim, it suffices to show that the complex $\operatorname{Syn}\left(R_{\alpha}, r\right)$ computes the rational geometric logsyntomic cohomology of $\mathscr{D}_{\alpha}:=\operatorname{Spf} \mathscr{O}^{+}\left(D_{\alpha}\right)$, the formal affine space over $\mathscr{O}_{C}$, that is a smooth formal model of $D_{\alpha}$. To do this, recall that the latter cohomology is computed by the complex

$$
\mathrm{R} \Gamma_{\text {syn }}\left(\mathscr{D}_{\alpha}, \mathbf{Z}_{p}(r)\right)_{\mathbf{Q}}=\left[\mathrm{R} \Gamma_{\text {cr }}\left(\mathscr{D}_{\alpha} / \mathrm{A}_{\text {cris }}\right)_{\mathbf{Q}}^{\varphi=p^{r}} \rightarrow \mathrm{R} \Gamma_{\text {cr }}\left(\mathscr{D}_{\alpha} / \mathrm{A}_{\text {cris }}\right)_{\mathbf{Q}} / F^{r}\right]
$$

where $A_{\text {cris }}$ is equipped with the unique log-structure extending the canonical logstructure on $\mathscr{O}_{C} / p$. It suffices thus to show that there exists a quasi-isomorphism $\mathrm{R} \Gamma_{\text {cr }}\left(\mathscr{D}_{\alpha} / \mathrm{A}_{\text {cris }}\right)_{\mathbf{Q}} \simeq \Omega_{R_{\alpha}}^{\bullet}$ that is compatible with the Frobenius ${ }^{(4)}$ and the filtration. But this is clear since $\operatorname{Spf} R_{\alpha}^{+}$is a log-smooth lifting of $\mathscr{D}_{\alpha}$ from $\operatorname{Spf} \mathscr{O}_{C}$ to $\operatorname{Spf} \mathrm{A}_{\text {cris }}$ that is compatible with the Frobenius on $\mathrm{A}_{\text {cris }}$ and $\mathscr{O}^{+}\left(D_{\alpha}\right) / p$.

To show the second claim, we note that, for $\beta \geq \alpha$, there is a natural map (an injection) of liftings $\left(R_{\beta}^{+} \rightarrow \mathscr{O}^{+}\left(D_{\beta}\right)\right) \rightarrow\left(R_{\alpha}^{+} \rightarrow \mathscr{O}^{+}\left(D_{\alpha}\right)\right)$. This allows us to use the comparison isomorphism (4) to define the second quasi-isomorphism in the sequence of maps

$$
\begin{aligned}
\tau_{\leq r} \mathrm{R} \Gamma_{\text {proét }}\left(\mathbf{A}^{n}, \mathbf{Q}_{p}(r)\right) & \simeq \tau_{\leq r} \operatorname{holim}_{k} \mathrm{R} \Gamma_{\text {ét }}\left(D_{k}, \mathbf{Q}_{p}(r)\right) \simeq \tau_{\leq r} \operatorname{holim}_{k} \operatorname{R} \Gamma_{\text {syn }}\left(\mathscr{D}_{k}, \mathbf{Z}_{p}(r)\right)_{\mathbf{Q}} \\
& \simeq \tau_{\leq r} \operatorname{holim}{ }_{k} \operatorname{Syn}\left(R_{k}, r\right)=\tau_{\leq r} \operatorname{Syn}\left(\mathbf{A}^{n}, r\right) .
\end{aligned}
$$

4. Recall that the Frobenius on crystalline cohomology is defined via the isomorphism $\mathrm{R} \Gamma_{\mathrm{cr}}\left(\mathscr{D}_{\alpha} / \mathrm{A}_{\text {cris }}\right)_{\mathbf{Q}} \stackrel{\sim}{\rightarrow} \mathrm{R} \Gamma_{\mathrm{cr}}\left(\left(\mathscr{D}_{\alpha}, / p\right) / \mathrm{A}_{\text {cris }}\right)_{\mathbf{Q}}$ from the canonical Frobenius on the second term. 
Here, the first quasi-isomorphism follows from the fact that $\left\{D_{k}\right\}_{k \in \mathbf{N}}$ is an admissible affinoid covering of $\mathbf{A}^{n}$ and the third one follows from the first claim. This finishes the proof.

\section{Computation of $\operatorname{HK}_{r}^{i}\left(\mathbf{A}^{n}\right)$}

The group $\operatorname{HK}_{r}^{i}\left(\mathbf{A}^{n}\right)$ is, by construction, obtained from the $\operatorname{HK}_{r}^{i}\left(R_{\alpha}\right)$ 's, but the latter are, individually, hard to compute and quite nasty: for example, $\operatorname{HK}_{1}^{1}\left(R_{\alpha}\right)$ is isomorphic to the quotient of $\mathbf{Q}_{p} \widehat{\otimes} \mathscr{O}\left(D_{\alpha}\right)^{*}$ by the sub $\mathbf{Q}_{p}$-vector space generated by $\mathscr{O}\left(D_{\alpha}\right)^{*}$; hence it is an infinite dimensionnal topological $\mathbf{Q}_{p}$-vector space in which 0 is dense. Fortunately Lemma 8 below shows that this is not a problem for the computation of $\operatorname{HK}_{r}^{i}\left(\mathbf{A}^{n}\right)$.

For $\mathbf{k}=\left(k_{1}, \ldots, k_{n}\right) \in \mathbf{N}^{n}$, we set $|\mathbf{k}|=k_{1}+\cdots+k_{n}$ and $T^{\mathbf{k}}=T_{1}^{k_{1}} \cdots T_{n}^{k_{n}}$. For $1 \leq j \leq n$, let $\omega_{j}$ be the differential form $\frac{d T_{j}}{T_{j}}$, and let $\partial_{j}$ be differential operator defined by $d f=\sum_{j=1}^{n} \partial_{j} f \omega_{j}$. For $\mathbf{j}=\left\{j_{1}, \ldots, j_{i}\right\}$, with $j_{1} \leq j_{2} \leq \cdots \leq j_{i}$, we set $\omega_{\mathbf{j}}=\omega_{j_{1}} \wedge \cdots \wedge \omega_{j_{i}}$. All elements $\eta$ of $\Omega_{R_{\alpha}}^{i}$ can be written, in a unique way, in the form $\sum_{|\mathbf{j}|=i} a_{\mathbf{j}} \omega_{\mathbf{j}}$, where $a_{\mathbf{j}} \in\left(\prod_{j \in \mathbf{j}} T_{j}\right) R_{\alpha}$.

Lemma 7. - Let $M$ be a sub- $\mathbf{Z}_{p}$-module of $\mathrm{A}_{\text {cris }}$ or $\mathscr{O}_{C}$. Let $i \geq 1$ and $\mathbf{k} \in \mathbf{N}^{n}$. For $\omega=T^{\mathbf{k}} \sum_{|\mathbf{j}|=i} a_{\mathbf{j}} \omega_{\mathbf{j}}$, with $a_{\mathbf{j}} \in M$, such that $d \omega=0$, there exists $\eta=T^{\mathbf{k}} \sum_{|\mathbf{j}|=i-1} b_{\mathbf{j}} \omega_{\mathbf{j}}$, such that $d \eta=\omega$ and $b_{\mathbf{j}} \in p^{-N(\mathbf{k})} M$, with $N(\mathbf{k})=\inf _{j \in \mathbf{j}} v_{p}\left(k_{j}\right)$.

Proof. - Permuting the $T_{m}$ 's, we can assume that $v_{p}\left(k_{1}\right) \leq v_{p}\left(k_{2}\right) \leq \cdots \leq v_{p}\left(k_{n}\right)$; in particular, $k_{1} \neq 0$. Decompose $\omega$ as $\left(\omega_{1} \wedge T^{\mathbf{k}} \sum_{1 \in \mathbf{j}} a_{\mathbf{j}} \omega_{\mathbf{j} \backslash\{1\}}\right)+\omega^{\prime}$, and set $\eta=$ $\frac{1}{k_{1}} T^{\mathbf{k}} \sum_{1 \in \mathbf{j}} a_{\mathbf{j}} \omega_{\mathbf{j} \backslash\{1\}}$; we have $\omega-d \eta=T^{\mathbf{k}} \sum_{1 \notin \mathbf{j}} c_{\mathbf{j}} \omega_{\mathbf{j}}$ and it has a trivial differential. But $d\left(T^{\mathbf{k}} \sum_{1 \notin \mathbf{j}} c_{\mathbf{j}} \omega_{\mathbf{j}}\right)=k_{1} T^{\mathbf{k}} \sum_{1 \notin \mathbf{j}} c_{\mathbf{j}} \omega_{\{1\} \cup \mathbf{j}}+\sum_{1 \notin I} c_{I}^{\prime} \omega_{I}$, hence $c_{\mathbf{j}}=0$ for all $\mathbf{j}$, which proves that $d \eta=\omega$ and allows us to conclude.

Lemma 8. - Let $\alpha \in \mathbf{Q}_{+}$and let $\Lambda_{\alpha}=R_{\alpha}^{+}, \mathscr{O}^{+}\left(D_{\alpha}\right)$. Then $H_{\mathrm{dR}}^{0}\left(\Lambda_{\alpha}\right)=\mathrm{A}_{\text {cris }}, \mathscr{O}_{C}$ and $\operatorname{HK}_{r}^{0}\left(R_{\alpha}^{+}\right)=\mathrm{A}_{\text {cris }}^{\varphi=p^{r}}$, the natural maps

$$
H_{\mathrm{dR}}^{i}\left(\Lambda_{\alpha+1}\right) \rightarrow H_{\mathrm{dR}}^{i}\left(\Lambda_{\alpha}\right), \quad i \geq 1 ; \quad \operatorname{HK}_{r}^{i}\left(R_{\alpha+2}^{+}\right) \rightarrow \operatorname{HK}_{r}^{i}\left(R_{\alpha}^{+}\right), \quad i \geq 2 .
$$

are identically zero, and the image of the $\operatorname{map} \operatorname{HK}_{r}^{1}\left(R_{\alpha+2}^{+}\right) \rightarrow \operatorname{HK}_{r}^{1}\left(R_{\alpha}^{+}\right)$is annihilated by $p^{r}$.

Proof. - The computation of the $H^{0}$ 's is straightforward. The proof for the first map is similar (but easier) to that of the second one, so we are only going to prove the latter. Take $i \geq 2$. Let $\left(\omega^{i}, \omega^{i-1}\right)$ be a representative of an element of $\operatorname{HK}_{r}^{i}\left(R_{\alpha+2}^{+}\right)$. That is to say $\omega^{i} \in \Omega_{R_{\alpha+2}^{i}}^{i}, \omega^{i-1} \in \Omega_{R_{\alpha+2}^{+}}^{i-1}, d \omega^{i}=0$ and $d \omega^{i-1}+\left(\varphi-p^{r}\right) \omega^{i}=0$.

Since $d \omega^{i}=0$, we deduce from Lemma 7 that there exists $\eta^{i-1} \in \Omega_{R_{\alpha+1}^{+}}^{i-1}$ such that $\iota_{\alpha+2, \alpha+1} \omega^{i}=d \eta^{i-1}$ (we used here that $\frac{1}{m}[\tilde{p}]^{m} \in \mathrm{A}_{\text {cris }}$ ). Let $\omega_{1}^{i-1}=\iota_{\alpha+2, \alpha+1} \omega^{i-1}+$ 
$\left(\varphi-p^{r}\right) \eta^{i-1}$. Then $d \omega_{1}^{i-1}=\iota_{\alpha+2, \alpha+1} d \omega^{i-1}+\left(\varphi-p^{r}\right) d \eta^{i-1}=0$; hence there exists $\eta^{i-2} \in \Omega_{R_{\alpha}^{+}}^{i-2}$ such that $\iota_{\alpha+1, \alpha} \omega_{1}^{i-1}=d \eta^{i-2}$. It follows that $\iota_{\alpha+2, \alpha}\left(\omega^{i}, \omega^{i-1}\right)=$ $d\left(\iota_{\alpha+1, \alpha} \eta^{i-1}, \eta^{i-2}\right)$, as wanted.

Take now $i=1$ and use the notation from the above computation. Arguing as above we show that $\left(\omega^{1}, \omega^{0}\right)$ is in the same class as $\left(0, \omega^{0}\right)$, with $\omega^{0} \in \mathrm{A}_{\text {cris }}$. But the map $\varphi-p^{r}: \mathrm{A}_{\text {cris }} \rightarrow \mathrm{A}_{\text {cris }}$ is $p^{r}$-surjective. This proves the last statement of the lemma.

Remark 9. - (i) The same arguments would prove that there exists $N: \mathbf{Q}_{+}^{*} \rightarrow \mathbf{N}$ such that, if $\beta>\alpha$ and $i \geq 1$, the images of the natural maps $H_{\mathrm{dR}}^{i}\left(R_{\beta}^{+}\right) \rightarrow H_{\mathrm{dR}}^{i}\left(R_{\alpha}^{+}\right)$, $\operatorname{HK}_{r}^{i}\left(R_{\beta}^{+}\right) \rightarrow \operatorname{HK}_{r}^{i}\left(R_{\alpha}^{+}\right)$are killed by $p^{N(\beta-\alpha)}$. This is sufficient to extend Corollary 10 and Lemma 11 to the unit ball $\stackrel{\circ}{ }^{n}$.

(ii) Note, however, that $N(u) \rightarrow+\infty$ when $u \rightarrow 0^{+}$. This prevents the extension of Lemma 11 to the integral de Rham cohomology of $\stackrel{\circ}{\mathbf{B}}^{n}$ which is good since this integral de Rham cohomology, in degrees $1 \leq i \leq n$, is far from 0 (but its $p$-torsion is dense).

Corollary 10. - If $i \geq 1$ then $\operatorname{HK}_{r}^{i}\left(\mathbf{A}^{n}\right)=0$.

Proof. - Immediate from Lemma 8 and the exact sequence

$$
0 \rightarrow \mathrm{R}^{1} \lim _{\overleftarrow{k}} \operatorname{HK}_{r}^{i-1}\left(R_{k}\right) \rightarrow \operatorname{HK}_{r}^{i}\left(\mathbf{A}^{n}\right) \rightarrow \underset{\leftarrow}{\lim _{k}} \operatorname{HK}_{r}^{i}\left(R_{k}\right) \rightarrow 0
$$

\section{Computation of $\operatorname{DR}_{r}^{i}\left(\mathbf{A}^{n}\right)$}

Lemma 11. - If $1 \leq i \leq r-1$ then $\operatorname{DR}_{r}^{i}\left(\mathbf{A}^{n}\right) \simeq\left(\Omega^{i}\left(\mathbf{A}^{n}\right) / \operatorname{Ker} d\right)(r-i-1)$, if $i \geq r$ then $\mathrm{DR}_{r}^{i}\left(\mathbf{A}^{n}\right)=0$, and, if $r>0$, we have an exact sequence

$$
0 \rightarrow \mathrm{B}_{\text {cris }}^{+} / F_{\theta}^{r} \rightarrow \operatorname{DR}_{r}^{0}\left(\mathbf{A}^{n}\right) \rightarrow\left(\mathscr{O}\left(\mathbf{A}^{n}\right) / C\right)(r-1) \rightarrow 0
$$

Proof. - We have an exact sequence

$$
0 \rightarrow \mathrm{R}^{1} \underset{k}{\lim _{k}} \mathrm{DR}_{r}^{i-1}\left(R_{k}\right) \rightarrow \mathrm{DR}_{r}^{i}\left(\mathbf{A}^{n}\right) \rightarrow \underset{k}{\lim _{k}} \mathrm{DR}_{r}^{i}\left(R_{k}\right) \rightarrow 0
$$

The $\operatorname{DR}_{r}^{i}\left(R_{k}\right)$ 's are the cohomology groups of the complex

$$
\ldots \longrightarrow \mathrm{A}_{r-i} \otimes \otimes_{\mathrm{A}_{\text {cris }}} \Omega_{R_{k}}^{i} \stackrel{1 \otimes d_{i}}{\longrightarrow} \mathrm{A}_{r-i-1} \otimes_{\mathrm{A}_{\text {cris }}} \Omega_{R_{k}}^{i+1} \longrightarrow \cdots
$$

In particular, they are trivially 0 if $i \geq r$, so assume $i \leq r-1$. The kernel of $1 \otimes d_{i}$ is $F_{\theta}^{r-i-1} \mathrm{~A}_{r-i} \otimes \mathrm{A}_{\text {cris }} \Omega_{R_{k}}^{i}+\mathrm{A}_{r-i} \otimes_{\mathrm{A}_{\text {cris }}}\left(\Omega_{R_{k}}^{i}\right)_{d=0}$ while the image of $1 \otimes d_{i-1}$ is $\mathrm{A}_{r-i} \otimes_{\mathrm{A}_{\text {cris }}} d \Omega_{R_{k}}^{i-1}$. Since $F_{\theta}^{r-i-1} \mathrm{~A}_{r-i}$ is an $\mathscr{O}_{C}$-module of rank 1 (generated by the image of $\left.\frac{\left(p-[\tilde{p})^{r-i-1}\right.}{(r-i-1) !}\right)$, we have $F_{\theta}^{r-i-1} \mathrm{~A}_{r-i} \otimes_{\mathrm{A}_{\text {cris }}} \Omega_{R_{k}}^{i} \simeq \Omega^{i}\left(D_{k}\right)(r-i-1)$, which gives us the exact sequence

$$
0 \rightarrow \mathrm{A}_{r-i} \otimes_{\mathrm{A}_{\text {cris }}} H_{\mathrm{dR}}^{i}\left(R_{k}\right) \rightarrow \mathrm{DR}_{r}^{i}\left(R_{k}\right) \rightarrow\left(\Omega^{i}\left(D_{k}\right) / \text { Ker } d\right)(r-i-1) \rightarrow 0 .
$$


For $i=0$ this gives the sequence in the lemma.

Assume that $i \geq 1$. The natural map $H_{\mathrm{dR}}^{i}\left(R_{k+1}\right) \rightarrow H_{\mathrm{dR}}^{i}\left(R_{k}\right)$ is identically zero by Lemma 8 . Hence

$$
\mathrm{R}^{j} \underset{k}{\lim _{k}}\left(\Omega^{i}\left(D_{k}\right) / \operatorname{Ker} d\right) \simeq \mathrm{R}^{j} \underset{k}{\lim _{k}} \mathrm{DR}_{r}^{i}\left(R_{k}\right), \quad j \geq 0 .
$$

Now, we note that since our systems are indexed by $\mathbf{N}, \mathrm{R}^{j} \varliminf_{k}$ is trivial for $j \geq 2$. Since $\mathrm{R}^{1} \varliminf_{k} \Omega^{i}\left(D_{k}\right)=0$, we have $\mathrm{R}^{1} \varliminf_{k}\left(\Omega^{i}\left(D_{k}\right) /\right.$ Ker $\left.d\right)=0$ (and $\mathrm{R}^{1} \varliminf_{k} d \Omega^{i}=0$ ). It remains to show that $\lim _{k}\left(\Omega^{i}\left(D_{k}\right) / \operatorname{Ker} d\right) \simeq \Omega^{i}\left(\mathbf{A}^{n}\right) / \operatorname{Ker} d$. But this amounts to showing that $\mathrm{R}^{1} \varliminf_{k} \Omega^{i}\left(\overleftarrow{D}_{k}\right)_{d=0}=0$. This is clear for $i=0$ and for $i>0$, since the system $\left\{H_{\mathrm{dR}}^{i}\left(R_{k}\right)\right\}_{k \in \mathbf{N}}$ is trivial (by Lemma 8), this follows from the fact that $\mathrm{R}^{1} \lim _{k} d \Omega^{i-1}\left(D_{k}\right)=0$.

\section{Proof of Theorem 1 and Theorem 3}

4.1. Algebraic isomorphism. - From Proposition 6 we know that $\tau_{\leq r} \operatorname{Syn}\left(\mathbf{A}^{n}, r\right) \simeq$ $\tau_{\leq r} \mathrm{R} \Gamma_{\text {proét }}\left(\mathbf{A}^{n}, \mathbf{Q}_{p}(r)\right)$. From the long exact sequence

$$
\cdots \rightarrow \operatorname{DR}_{r}^{i-1}\left(\mathbf{A}^{n}\right) \rightarrow \operatorname{Syn}^{i}\left(\mathbf{A}^{n}, r\right) \rightarrow \operatorname{HK}_{r}^{i}\left(\mathbf{A}_{n}\right) \rightarrow \operatorname{DR}_{r}^{i}\left(\mathbf{A}^{n}\right) \rightarrow \cdots
$$

and Corollary 10 and Lemma 11, we obtain isomorphisms

$$
\left(\Omega^{i-1}\left(\mathbf{A}^{n}\right) / \operatorname{Ker} d\right)(r-i) \stackrel{\sim}{\rightarrow} \operatorname{Syn}^{i}\left(\mathbf{A}^{n}, r\right), \quad r \geq i \geq 2,
$$

and the exact sequence

$$
0 \rightarrow \operatorname{Syn}^{0}\left(\mathbf{A}^{n}, r\right) \rightarrow \mathrm{B}_{\text {cris }}^{+, \varphi=p^{r}} \rightarrow \operatorname{DR}_{r}^{0}\left(\mathbf{A}^{n}\right) \rightarrow \operatorname{Syn}^{1}\left(\mathbf{A}^{n}, r\right) \rightarrow 0,
$$

which, using the fundamental exact sequence

$$
0 \rightarrow \mathbf{Q}_{p}(r) \rightarrow \mathrm{B}_{\text {cris }}^{+, \varphi=p^{r}} \rightarrow \mathrm{B}_{\text {cris }}^{+} / F_{\theta}^{r} \rightarrow 0
$$

proves the first isomorphism in Theorem 1 (together with $\operatorname{Syn}^{0}\left(\mathbf{A}^{n}, r\right) \cong \mathbf{Q}_{p}(r)$ ). The second isomorphism is an immediate consequence of the fact that $H_{\mathrm{dR}}^{i}\left(\mathbf{A}^{n}\right)=0$.

Since an open ball is an increasing union of closed balls, Theorem 3 is proved by the same argument (see Remark 9).

Remark 12. - (i) Let $j \in \mathbf{N}$. We note that, since $[\tilde{p}]^{p} \in p \mathrm{~A}_{\text {cris }}$, for every $\alpha \in \mathbf{Q}_{+}$, the maps ${ }^{(5)} \Omega^{i}\left(R_{\alpha+m}^{+}\right)_{j} \rightarrow \Omega^{i}\left(R_{\alpha}^{+}\right)_{j}, m \geq p j$, are the zero maps for $i \geq 1$ and the projection on the constant term for $i=0$. It follows that

$$
\operatorname{holim}_{k} \operatorname{HK}_{r}\left(R_{k}^{+}\right)_{j} \simeq\left(\mathrm{A}_{\text {cris }, j} \stackrel{\varphi-p^{r}}{\longrightarrow} \mathrm{A}_{\text {cris }, j}\right), \quad \operatorname{holim}_{k} \mathrm{DR}_{r}\left(R_{k}^{+}\right)_{j} \simeq\left(\mathrm{A}_{\text {cris }} / F_{\theta}^{r}\right)_{j} .
$$

Computing as above we get $\left(\operatorname{holim}_{k, \ell} \operatorname{Syn}\left(R_{k}^{+}, r\right)_{j}\right) \otimes \mathbf{Q} \simeq \mathbf{Q}_{p}(r)$. Hence, by the comparison isomorphism (4), $H_{\text {êt }}^{i}\left(\mathbf{A}^{n}, \mathbf{Q}_{p}(r)\right)=0, i \geq 1$, which allows us to recover the result of Berkovich [1].

5. The subscript $j$ refers to moding out by $p^{j}$. 
(ii) The above argument does not go through for the open unit ball: the integral de Rham complex does not reduce to the constants in that case and $H_{\text {êt }}^{i}\left(\stackrel{\circ}{B}^{n}, \mathbf{Q}_{p}(r)\right)$ is an infinite dimensionnal $\mathbf{Q}_{p}$-vector space if $1 \leq i \leq n$.

4.2. Topological considerations. - It remains to discuss topology. In what follows, we write $\cong$ for an isomorphism of vector spaces and $\equiv$ for an isomorphism of topological vector spaces.

First, note that all the cohomology groups under consideration are cohomology groups of complexes of Fréchet spaces (and even of finite sums of countable products of Banach spaces), since these complexes can be built out of Čech complexes coming from coverings by affinoids, and the corresponding complexes for affinoids involve finitely many Banach spaces. It follows that, a priori, all the groups we are dealing with are cokernels of maps $F_{1} \rightarrow F_{2}$ between Fréchet spaces. If such a group injects continuously into a Fréchet space, then it is a Fréchet space (it is separated hence the image of $F_{1}$ in $F_{2}$ is closed, and our space is a quotient of a Fréchet space by a closed subspace), and if this injection is a bijection then it is an isomorphism of Fréchet spaces by the Open Mapping Theorem.

Now, we have the following commutative diagram:

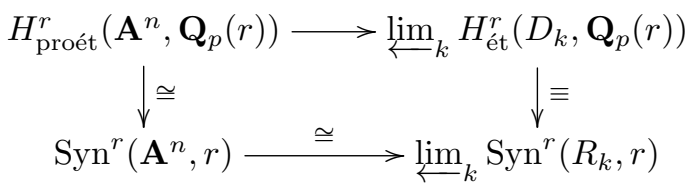

The horizontal maps are the natural maps (and are continuous), the bottom one being an isomorphism by the earlier computations. The left vertical arrow is an isomorphism by Proposition 6 and the right vertical arrow is a topological isomorphism because the period maps (4) are $p^{N}$-quasi-isomorphisms, with $N$ depending only on $r$. Thus proving that $\lim _{k} \operatorname{Syn}^{r}\left(R_{k}, r\right)$ is Fréchet would imply that so is $H_{\text {proét }}^{r}\left(\mathbf{A}^{n}, \mathbf{Q}_{p}(r)\right)$ and that $H_{\text {proét }}^{r}\left(\mathbf{A}^{n}, \mathbf{Q}_{p}(r)\right) \equiv \lim _{k} \operatorname{Syn}^{r}\left(R_{k}, r\right)$.

For that, consider the map of distinguished triangles

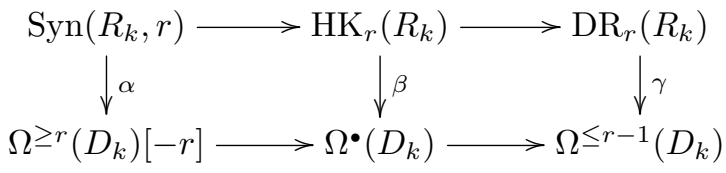

in which:

- the top line is the definition of $\operatorname{Syn}\left(R_{k}, r\right)$, the bottom one is the obvious one,

- $\gamma$ is obtained by applying $\theta$ to the terms of the complex $\operatorname{DR}_{r}\left(R_{k}\right)$,

- $\beta$ is obtained by composing the natural map $\operatorname{HK}_{r}\left(R_{k}\right) \rightarrow \Omega_{R_{k}}^{\circ}$ with $\theta$,

- $\alpha$ is obtained by composing the natural map $\operatorname{Syn}\left(R_{k}, r\right) \rightarrow F^{r} \Omega_{R_{k}}^{\bullet}$ with $\theta$. 
All the maps are continuous (including the boundary maps). For $r \geq 2$, taking cohomology and limits we obtain the commutative diagram

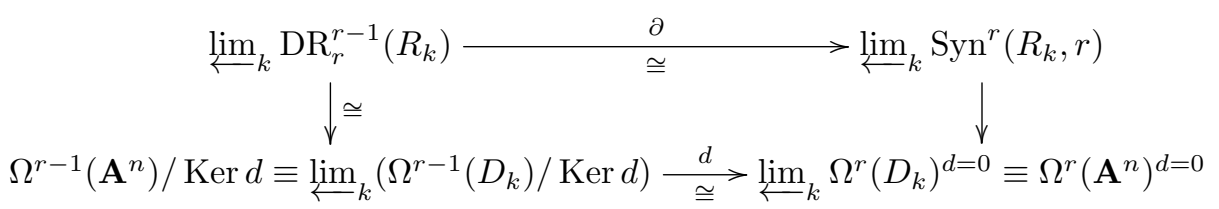

The bottom map is an isomorphism because $\varliminf_{k} H_{\mathrm{dR}}^{r}\left(D_{k}\right) \simeq H_{\mathrm{dR}}^{r}\left(\mathbf{A}^{n}\right)=0$. The top map is an isomorphism because, on level $k$, its kernel and cokernel are controlled by $\operatorname{HK}_{r}^{r-1}\left(R_{k}\right)$ and $\operatorname{HK}_{r}^{r}\left(R_{k}\right)$ respectively, which die in $R_{k-2}$ by Lemma 8 , and the left vertical map is an isomorphism by the proof of Lemma 11. The space $\Omega^{r}\left(\mathbf{A}^{n}\right)$ is Fréchet; it follows that all other spaces are also Fréchet (in particular $\varliminf_{k} \operatorname{Syn}^{r}\left(R_{k}, r\right)$ ) and that all the maps are topological isomorphisms. This concludes the proof of Theorem 1 if $r \geq 2$.

For $r=1$, the argument is similar, with $\lim _{k} \operatorname{DR}_{r}^{r-1}\left(R_{k}\right)$ in the above diagram replaced by $\left(\lim _{k} \mathrm{DR}_{r}^{r-1}\left(R_{k}\right)\right) / C$.

The proof for the open ball is similar.

\section{References}

[1] V. Berkovich, On the comparison theorem for étale cohomology of non-Archimedean analytic spaces, Israel J. Math. 92 (1995), 45-59.

[2] P. Colmez, G. Dospinescu, W. Nizioł, Cohomology of p-adic Stein spaces, arXiv:1801.06686 [math.NT].

[3] P. Colmez, W. Nizioł, Syntomic complexes and p-adic nearby cycles, Invent. Math. 208 (2017), 1-108.

[4] J.-M. Fontaine and W. Messing, p-adic periods and p-adic étale cohomology, Current Trends in Arithmetical Algebraic Geometry (K. Ribet, ed.), Contemporary Math., vol. 67, AMS, Providence, 1987, 179-207. Math. Z. 247 (2004), 223-240.

[5] O. Hyodo, K. Kato, Semi-stable reduction and crystalline cohomology with logarithmic poles. Astérisque 223 (1994), 221-268.

[6] A-C. Le Bras, Espaces de Banach-Colmez et faisceaux cohérents sur la courbe de FarguesFontaine, arXiv:1801.00422 [math.NT].

[7] T. Tsuji, p-adic étale cohomology and crystalline cohomology in the semi-stable reduction case, Invent. Math. 137 (1999), 233-411.

Pierre Colmez, C.N.R.S., IMJ-PRG, Sorbonne Université, 4 place Jussieu, 75005 Paris, France E-mail : pierre.colmez@imj-prg.fr

WiesŁawa NizioŁ, CNRS, UMPA, École Normale Supérieure de Lyon, 46 allée d'Italie, 69007 Lyon, France - E-mail : wieslawa.niziol@ens-lyon.fr 